\title{
Ānāpāna: Neuro-engineering of Mindfulness
}

\author{
Ānāpāna: Neuroingeniería de la Meditación
}

\author{
Carlos Diego Ferrin Bolaños, Esteban Mauricio Correa Agudelo, Andrés Mauricio Hernández \\ Camacho, Juan Diego Gómez \\ Centro de Bioinformática y Biología Computacional de Colombia, Manizales, Colombia \\ correo-e: carlos.ferrinabios.co \\ esteba.correa@bios.co \\ andres.hernandez@bios.co \\ juan.gomez@bios.co
}

\begin{abstract}
Meditation benefits have been widely supported by many research works. It is considered a natural way of reducing stress, depression, and other anxiety disorders. However, meditation process is a breathe pursuing task that requires long time training to avoid the so-called mind wandering. In this work, we propose a neural-feedback system that detects when mind wanders and generates an audible signal, this way patient's attention is recovered back again. Finally, we support our approach by testing the system on novice and advanced meditators.
\end{abstract}

Key Word - Machine Learning, Electroencephalography (EEG), Neurofeedback, Functional Magnetic Resonance (fMRI), Psychotherapy, Signal Processing.

Resumen-Los beneficios psicoterapéuticos de la meditación han sido avalados científicamente en muchos trabajos a nivel mundial, considerándola una forma natural de reducir los niveles de estrés, depresión, etc. Sin embargo, la meditación mediante el seguimiento consciente de la respiración es un proceso que requiere largos periodos de entrenamiento para aprender a evitar la denominada desviación de la atención. En este trabajo se propone un sistema de retroalimentación neuronal que detecta la desviación de la atención y genera una señal auditiva que recupera la atención del paciente. A continuación, se reportan resultados preliminares que avalan el enfoque propuesto en este trabajo.

Palabras clave- Máquinas de Aprendizaje, Electroencefalografía (EEG), Resonancia Magnética Funcional (fMRI), Psicoterapia, Procesamiento de Señales.

\section{INTRODUCTION}

Depression is a mental disorder with a profound negative effect on behavior and quality of life. According to the World Health Organization, in about 20 years, depression will be the disorder with the most significant repercussions, both socially and economically [1]. Recently, fundamental and applied research has investigated purported benefits of meditation and mental training. Foremost, neuroscience has focused on the brain mechanisms and neural correlates of meditation. Particularly, R. Davidson et al [10] have been leading a series of uncommon, yet seminal studies involving Buddhists monks [11], [8], [12], [13], [20]. For instance, groundbreaking experiments conducted with French Buddhists monk Matthieu Ricard yielded recently remarkable results: "during meditation, Ricard's brain produces a level of gamma waves those linked to consciousness, attention, learning and memory - never reported before in the neuroscience literature" [11]. Inherently, of course, computer vision, signal processing, machine learning and pattern recognition, they all have contributed to achieve these findings in more technical aspects. In sight of this, nowadays, few doubt about the benefits of mindfulness meditation in treating mental disorders [14], [15], [22], [24], and [26]. This particularly applies to depression [14], [17], a psychological condition that is treated, otherwise, with several different invasive approaches such as antidepressant drugs [31], transcranial stimulation [32] and more recently, Araujo et al [33] have used psychedelic plants such as Ayahuasca.

Despite the scientifically proved benefits of meditation and its potential use for healing medical conditions. At the best of our knowledge, nobody has tried yet to engineer a system that permits automatic learning of mindfulness meditation, as an aid for trainees and patients. Therefore, the introduction, design and prototype of such a system are the main contributions of this paper. To start with, we argue three scientific facts that will endorse our approach:

- Depression is increased by Mind-wandering: Mindwandering is the experience of constantly changing thoughts, without any awareness of doing so. This happens particularly when we are not engaged in an attention-demanding or goal-directed task (Fig.1, left). While mind wandering may be helpful sometimes, (e.g. it is linked to creativity), when excessive it may bring devastating consequences. This is mainly the case because most of the time, we 
mind-wander to stressful thoughts such as worries, ruminations, pre-occupations etc. In fact, scientific evidence amply supports that mind wandering is positively correlated with the severity of depression. More precisely, a major depression disorder follows an increase of mind-wandering and task-unrelated thoughts. [2], [14], [24], [25], [26], [28], [29].

- Mind wandering arises from activity in the DefaultMode Network (DMN): Recently, neuroscientists have found the neural basis of mind-wandering (Fig. 1 , middle). There is a network of brain regions (DMN) that oscillate synchronously and more active when the mind wanders relative to when engaged in attention-demanding tasks. Indeed, functional neuroimaging (fMRI) studies show that during wakeful rest, this network exhibits highest coherent neural activation. Likewise, task-induced deactivation was found possible when participants were asked to concentrate in goal-directed task. Overall, the DMN has been hypnotized to produce spontaneous thoughts during mind wandering. [3], [14], [16], [21], [23], [27], [30].

- Mindfulness meditation regulates DMN activity: Mindfulness is form of meditation meant to develop the skill of paying attention by being fully aware of inner and outer experiences. Not surprisingly, studies suggest that the neural mechanisms underlying mindfulness training are associated with differential activity of the DMN. Specifically, functional neuroimaging reveals that after a 40-day course, reduction of DMN activity in naïve meditators becomes apparent. Awareness of the breath is perhaps the most popular kind of mindfulness. Trainees must watch their breath attentively and uninterruptedly, as it occurs naturally (for as long as possible), see Fig. 1, right. This seemingly simple task turns out extremely difficult for those unfamiliar with the method, let alone depressed patients. No matter how hard they try, their minds constantly wander without awareness of doing so, instead of following the breath. [4], [15], [16], [17], [19], [22], [30].

Roughly, in our framework (Fig. 2) the patient needs to have his brain activity measured while practicing mindfulness (e.g. using an fMRI machine). By precisely assessing the activity of the DMN, the medical team (i.e. a neuroscientist or/and physiotherapist) will know whether the brain of the patient is engaged in meditation, or has entered a mind-wandering state. If the latter proves true, the patient will be gently reminded (by the therapist) to go back to his mediation, so the mind wandering will be immediately cut off. The key aspect here is the timely reminder, which is impossible in self-training, since mind wandering begins unconsciously. With sustained practice of this guided methodology (in a daily basis), we expect the patient to enhance his attention and lower his DMN activity in the end.
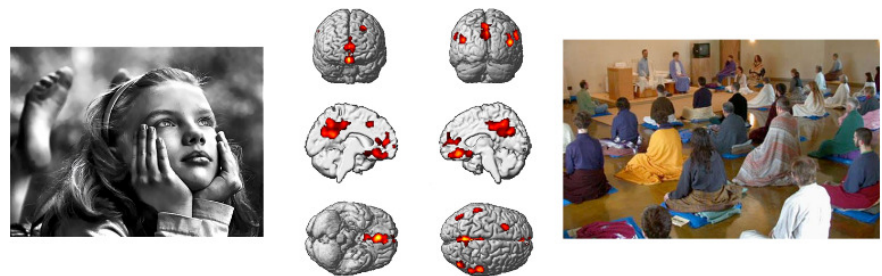

Figure 1. Left: mind-wandering girl. Middle: Areas of the brain involved in mind wandering (default mode network). Right: A group of trainees in mindfulness meditation.

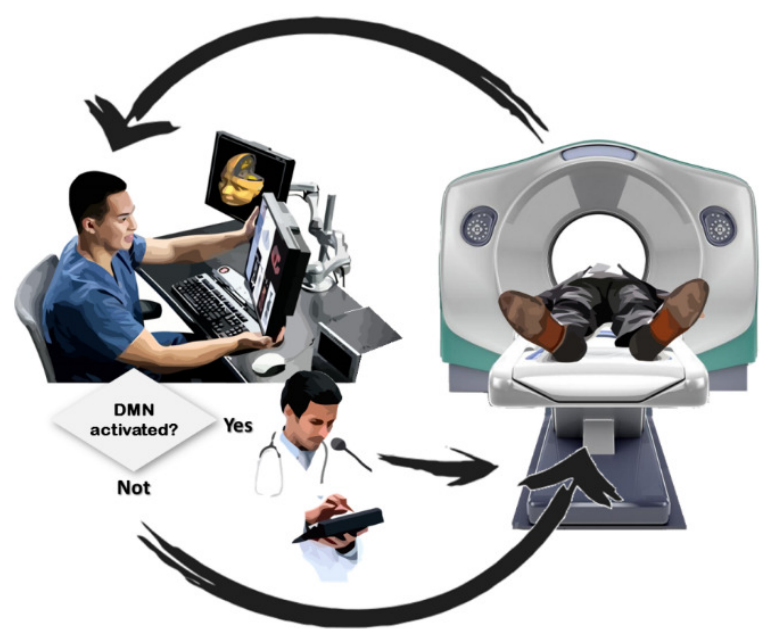

Figure 2. Computer-aided mindfulness meditation: Brain activity of a patient is assessed with an fMRI machine. If DMN activity is detected, a warning voice (microphone) alerts the patient. Otherwise, the cycle of assessment continues without intervention.

Although we think this methodology is bound to succeed, some disadvantages are apparent: the technology is unaffordable and the human resources are untenable for a daily therapy (mornings, afternoons and nights). There are also practical issues associated to this model: the position of the patient easily elicits sleepiness and the 'reminding' voice may break his concentration instead of holding it (frights are likely). In light of this, we put forward in what follows a smart prototype capable of executing the proposed methodology, while dramatically decreasing technology costs and removing human intervention.

\section{METHODOLOGY}

Overall, Ānāpāna is an assistive device that artificially enhances the breath if the mind wanders. The more the mind wanders, the more the breath is enhanced. In short, attention does not pursue breath, but breath pursues attention. To this end, we use three key elements to be described in this section: the telemindphone, the offsetting microphone and the super interface; see Fig. 3: 


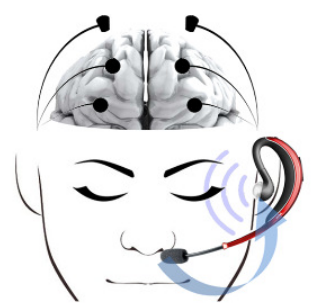

Figure 3. A graphical representation of our system Ānāpāna.

A. The telemindphone.

A low-cost, yet efficient approach to brain activity monitoring is a wireless EEG headset. A good example is the Emotiv EPOC, a high resolution, multi-channel, portable neuroheadset whose signal interpreter is typically a computer [7]. Although in our case, we transmit wirelessly EEG signals to an iPhone (not a computer), which natively runs a super smart interface that assesses the DMN activity. The coupling of these two technologies is what we term a telemindphone. In brief, we place the 14 data collecting electrodes and 2 reference electrodes of the Emotiv EPOC, in roughly international convention [5] with labels as shown in Fig. 4 and 6. The headset transmits encrypted data wirelessly to the phone whose wireless chip is proprietary and operates in the same frequency range as $802.11(2.4 \mathrm{Ghz})$. The connection is completed, once we are able to extract valuable signals (from the neuroheadset) through smart signal processing and classification algorithms running on the phone (super interface).

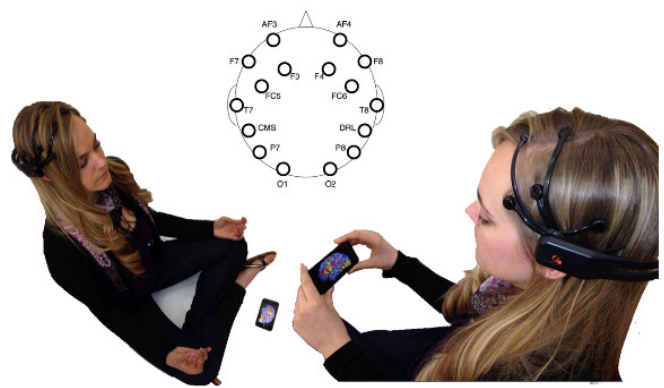

Figure 4. A telemindphone measuring brain activity (sides) and the connectivity protocol for EEG (top-center).

\section{B. The offsetting microphone.}

If we try to follow uninterruptedly our breath, at some point, we will suddenly realize that we had stopped doing so inadvertently, as we find ourselves having any sort of unrelated thoughts. It is as though the sound of the breath (in our mind) had vanished unnoticeably, when spontaneous thoughts arose and became louder. Thus, our idea (Fig. 5) is to regulate the loudness of the breath to keep it constant and unaffected by spontaneous thoughts (mind wandering). Specifically, we use an earphone to convey the sound of the breath (captured by a microphone attached to the nostrils) with a loudness that is proportional to the activity in the DMN (estimated by the telemindphone). For instance, if the DMN remains deactivated, no breath feedback will be transmitted. In contrast, when the DMN shows subtle activation so does the microphone to compensate the lack of attention. Accordingly, the sound of the breath never gets outshined by the wandering of the mind. This can be regarded as a gentlerise reminder for the patient to fix back his attention to the breath. Our hypothesis is that artificially enhanced breath prevents mind wandering. We advise against the use of an alarm (or a voice, Fig. 2) because this will simply break concentration: the therapeutic idea is not to resume concentration, but hold it for as long as possible.

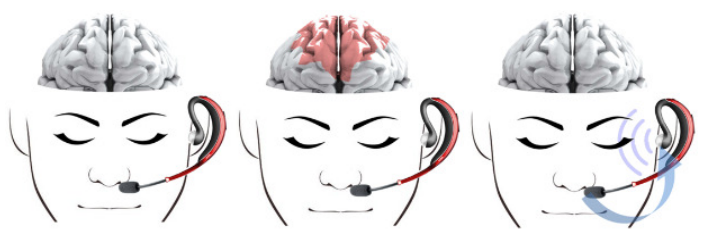

Figure 5. Meditation state (left). Mind-wandering state (middle), which correlates DMN activity (red stain). Meditation state recovered by the offsetting microphone progressive activation (right).

\section{The superinterface.}

Our interface is a multitask tool at the core of our system. It regulates the offsetting microphone through brain waves, and enriches the user experience with a sophisticated yet, very friendly interaction. There are three global features that remarkably define our interface in an overall picture: signal processing, auto regulation, and remote medical follow-up. This paper, although, offers insights just into the signal processing. Particularly, the extraction of patterns within brain waves using machine learning. This is the case because the two latter features (i.e. auto-regulation and remote follow up) lie out of the scope of this manuscript. More precisely, they belong in research fields rather distant to neurocomputing, namely software engineering, mobile interfaces and human computer interaction. Notwithstanding, we do describe them briefly at the end of this section as to provide a holistic portrait of Ānāpāna.

1) Intelligent signal processing: Evaluating brain activity in the DMN is known to be difficult, especially if an Emotiv EPOC is used instead of an fMRI machine. In fact, at the best of our knowledge, it has not been done yet. On the one hand, our interface has to deal with incomplete data, for the EEG connectivity is not dense at all. On the other hand, the Emotive is particularly prone to noise, which calls for an increasing of the Signal to Noise Ratio (SNR) using Independent Component Analysis (ICA) as described in [39], [40], [6]. More importantly though is the need for Machine Learning algorithms to classify patterns of DMN activity hidden within the EEG signals (Fig. 4). To this end, our interface uses Artificial Neural Networks (ANN) trained with deep learning algorithms [6], [34], and [37]. Accurate training of these ANNs requires also the gathering of fMRI datasets, which provide a baseline for learning and comparisons. We 
trained our ANNs in off-line mode as follow: we collected both negative and positive examples of DMN activation by asking first six well-trained meditators (teachers) to meditate inside an fMRI machine, while wearing the Emotiv EPOC. In like manner, we ask six other subjects not meditators, to simply rest under same conditions. Thus, we can gather fMRIs and corresponding EEGs of both, mindfulness and mindwandering states (Fig. 6). Once both groups get into performing their tasks, we evaluate them based on the fMRI images. Only if the DMN is indeed highly activated (not meditators) we proceed to record the EEG. Likewise, only when the DMN shows negligible activity (meditators) the EEG is also recorded. All subjects are asked to perform their respective tasks 10 times during at least one minute. Then, we record EEG and fMRI data for 30 seconds. Accordingly, we collect a total of 1800 seconds of EEG signals for each case: mindfulness and mind wandering. Finally, each second of these EEG signals serves as a pattern of DMN activity (positive or negative), when treated in the frequency domain. This makes a total data set of 3600 patterns for training.
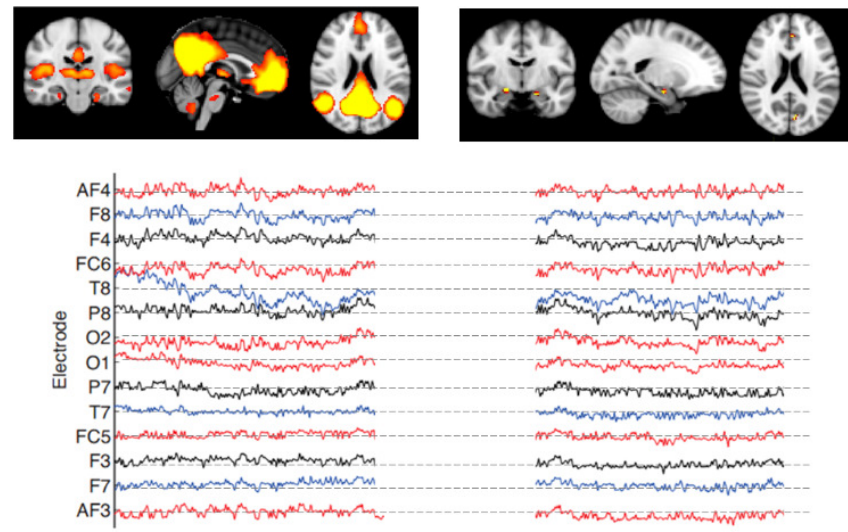

Figure 6. Two example of DMN activity described by fMRI images (top) and the corresponding EEG signals (bottom). Left: relatively high activity, which correlates a mind-wandering state (positive activation). Right: relatively low activity, which correlates a mindfulness state (negative activation). These one-second EEG signals are used as patterns to be learned by our ANN, once transformed to frequency domain. Refer to Fig. 4 to see electrode labeling and scalp mapping.

As mentioned, we use deep Neural Networks; i.e. networks with more than three layers. However, training these neural networks is known to be hard. The standard learning strategy (consisting of randomly initializing the weights of the network and applying gradient descent using backpropagation) is known empirically to find poor solutions for networks with three or more hidden layers. A clever strategy to train deep neural networks (known as deep learning) consists in simply initializing the weights (before applying gradient descent) not randomly but following a strategy. This strategy is rather simple: each layer but the last one, is trained individually as an unsupervised autoencoder [35], [38]. Here, an autoencoder is a one-layer neural network that applies backpropagation, setting the target values to be equal to the inputs. It is called unsupervised because the outputs need no labels, as they are simply the same inputs, which is not a condition in the general case of ANN. Once all the hidden layers have been trained, we apply standard supervised gradient-based learning to the whole network. This will affect both the pre-trained hidden layers (fine-tuning) and the untrained output layer (classification task). This pre-training strategy is known to improve on the traditional random initialization by providing "clues" to each intermediate layer about the kinds of representations that should be learnt, and thus initializing the supervised fine-tuning optimization in a region of parameter space from which a better local minimum of the error function can be reached. The overall structure of our network has been empirically set to 10 layers of 480, 400, 300, 150, 80, 40, 20, 10,5 and 1 neuron respectively.
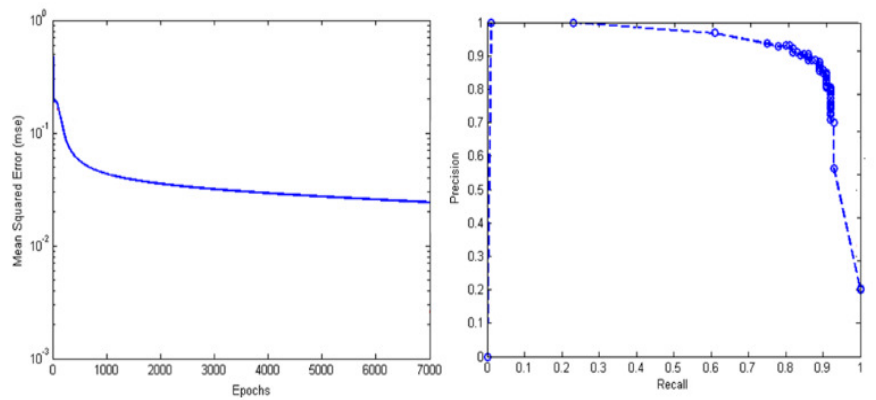

Figure 7. Performance of our ANN during training and validation. Left: training performance (goal error: 0.001; minimum error: 0.02424). Right: Precision-recall curve (best precision: 0.855; best recall 0.872 ). For training $70 \%$ of the collected data set was used; i.e. 2520 patterns. The remaining $30 \%$ was used for validation; i.e. 1080 patterns.

On the one hand, the size of the first layer obeys to the size of an input pattern. To arrange an input pattern we calculate the first 40 frequency components for each of the 14 one-second EEG signals (from AF4 to AF3, see Fig. 6). Next, we concatenate them all together to achieve a 480-dimensional vector. We use only 40 frequency components $(1-40 \mathrm{~Hz})$, because this is a widely known suitable range to study the most important EEG rhythmic activity frequency bands; i.e. delta, theta, alpha, beta etc. [16]. On the other hand, the last layer of the ANN has only one neuron that ultimately estimates how positive $(+1$, mind wandering), or negative $(-1$, mindfulness) the input vector is. Autonomously, we chose this layer not to be binary but real between -1 and +1 ; i.e. a tansigmoid transfer functions. Therefore, we can observe smooth transitions from mindfulness to mind-wandering states, and vice versa. In fact, the offsetting microphone is increasingly activated when the ANN output ranges from -0.5 up to +1 . The performance of this ANN is shown in the next figure (Fig. 7).

Note that the interface does not carry out the training, but simply host the tuned weights of the ANN. Once one-second EEG signal from the Emotive is received, preprocessed and converted to frequency domain. A multiplication between this signal and such weights takes place in order to calculate the 
ANN output. In what follows we want to outline the deep learning method we have used to train our ANN. Roughly, each layer (l) of the deep network is to be treated as an individual autoencoder [34], [38], by taking the previous layer (1-1) as input layer and adding it a temporal layer of the same size as output layer. After training, tuned weights of this temporal layer are dropped. In contrast, tuned weights between layers 1-1 and 1 are transferred, as the initial weights (between those layers), to the original network that is yet to be trained as a whole (fine-tuning). If we repeat this process layer by layer, we will be learning features of the input in the first layer, features of features in the second and so on. In other words, each layer is to encode a more abstract version of the input. The final layer of the original network that is meant to respond with the expected outputs of the global problem we are training for; will be tuned once the backpropagation ([6], [37]) algorithm is applied globally to the network. This routine is summarized in the pseudo-code shown in Table 1.

\begin{tabular}{|c|c|}
\hline 1. Ir & tialize $\mathrm{W}$ randomly; \\
\hline 2. $\%$ & re-training \\
\hline 3. $\mathrm{fc}$ & $i \in\{1 \ldots L-1\}$ do \\
\hline 4. & if $i==1$ \\
\hline 5. & Itemp=I; \\
\hline & else \\
\hline 7. & Itemp= tan-sigmoid(W(i)* Itemp); \\
\hline 8. & end \\
\hline 9. & Otemp=Itemp; \\
\hline $\begin{array}{l}10 . \\
\text { size }\end{array}$ & $\begin{array}{l}\text { create a ANNi of tree layers (size(Itemp), } \\
N(\text { i)), size(Otemp)); }\end{array}$ \\
\hline
\end{tabular}

11. train ANNi with Itemp and Otemp using standard backpropagation; 12. $\quad W(i)=W A N N i$ in hidden layer;

13. end

14. \%fine tuning phase

15. train the original ANN with I and O using standard backpropagation;

Table 1. Pseudo-code for Deep Learning ANNs.

$W$ represents the weights of the original network to be trained as a classifier in this thesis. $\boldsymbol{L}$ is the total number of layers of the network. $\boldsymbol{I}$ and $\boldsymbol{O}$ represent the inputs and outputs respectively or patterns to be learned in the classification problem. $\boldsymbol{W}(\boldsymbol{i})$ are the weights of the layer $\boldsymbol{i}$ in the global network. Itemp and Otemp are the temporary patterns to be learned by autoencoders-like hidden layer. Finally, note that a log-sigmoid function tan-sigmoid has been chosen as the activation function for all the neurons in this network.

2) Self-regulation: Our interface (Fig. 8) is highly configurable. Thus, based on the medical diagnosis, we can set up a daily routine for meditation (times, periods, repetitions, etc.) and take it with us wherever we go. Overall, this interface is an enriching new resource dedicated to providing patients with the tools and exercises they need in a format that is accessible and easy-to-use. Over 20 meditations, prayers, mantras and exercises are automatically played if stress (or anxiety) is detected. We can detect levels of stress using both the microphone activations (the more it is activated, less concentration is being kept). As for the microphone activation, it is optimal: at the beginning, the loudness of the first breaths is measured (in decibels). Thus, the interface knows how much the current breath sound needs to be amplified to keep loudness high enough, when mind wandering appears.

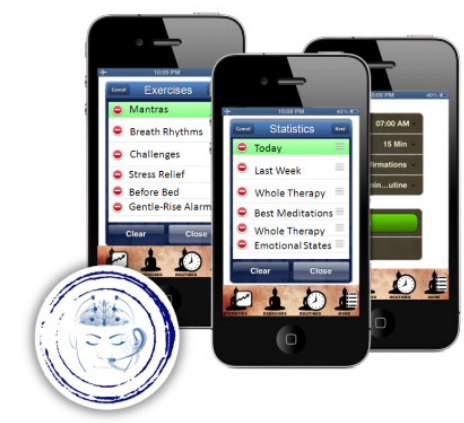

Figure 8. Examples of our superinterface.

The interface accounts for statistics, so it can tell whether a patient is meeting his medical prescription or not. If not, it reconfigures automatically all routines and times, so meditative goals are not missed within stipulated times. Further, speech-based reminders and advices are often given after practical sessions, such as the maximum period of uninterrupted concentration was 3.45 minutes, the next session won't be stopped until 5 concentration periods of 3 minutes have been reached', or 'an uninterrupted period of 10.4 minutes of concentration was registered in this section, that's twice as much as expected. The next section of this morning has been canceled, take a rest'. If wished, statistics can be automatically shared in social networks such as Facebook or Twitter. This is meant to create a collaborative network where participants can: challenge each other, evaluate their performance, find advises; or just to promote social interaction with peers, which is known to be good for overcoming depression.

3) Remote medical follow-up: This last feature (Fig. 9) aims at reducing costs of the treatment, while enhancing conformability and efficiency. Patients and clinics may save time and money related to geographical displacements. Let alone that depressed patients are generally reluctant to go outside home. Likewise, not treating a patient on time may also increase the cost in the end. Through our interface, patient and therapist remain linked $24 / 7$ in a point-to-point internet-based connection. The therapist can remotely follow the progresses, update routines, send private chats and monitor the emotional states of the patient as he practices; all this in real time. Needless to say, that this is a precious diagnostic tool to understand if a patient needs further treatment, or definitely the use of specific drugs is advisable. 
On the other side, the patient can use the private chat to ask for feedback, advises, encouragement or counseling, which are all helpful in nursing him back to health. Further, if needed, therapies may be held using video calling assistance.

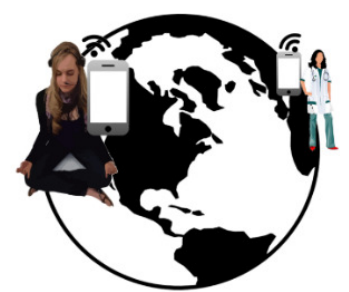

Figure 9. Remote medical follow-up.

\section{EXPERIMENTS AND RESULTS}

For this test we recruited 10 first-week trainees from a meditation course (novice meditators), whose age ranged from 23 to 44 . A first group ( 5 subjects) was asked to meditate for 5 minutes wearing the Emotiv, as we recorded their ANN outputs. Given that we can get an ANN output every second, we collected a total 1500 outputs, i.e. 60 seconds x 5 minutes $\mathrm{x} 5$ subjects. For this first group, the offsetting microphone was fully deactivated (i.e. meditation without the aid of Ānāpāna). Afterwards, a second group (5 subjects) was given 40 minute training about the experiment. Basically, they all were explained both, the central idea of Ānāpāna and how to react to the gentle-rise alarm of the microphone. Further, they themselves tried the system several times in order to get familiar with it. After that, we ran the second recording (1500 more ANN outputs), this time with the microphone on; i.e. meditation with the aid of Ānāpāna. The following boxplot (Fig. 10) shows the distributions of the ANN outputs for the each group of meditators during the five-minute test. Additionally, we also report a third group (as a baseline) of five experienced meditators (using Ānāpāna) recorded during five minutes of meditation as well (1500 more ANN outputs).

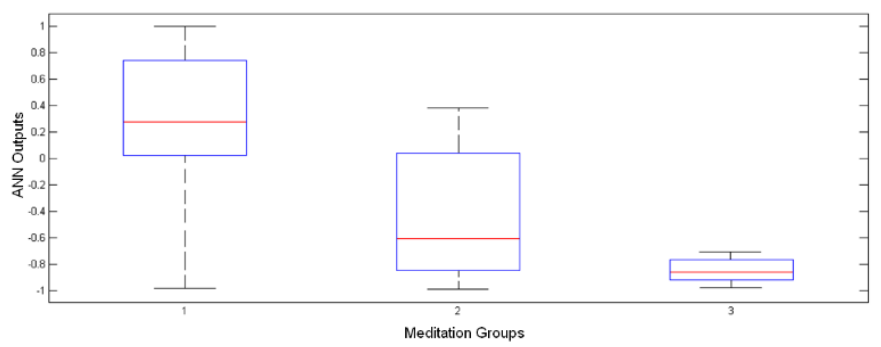

Figure 10. Boxplot quartiles distribution of the ANN outputs during our experiments.

In Fig. 10, we see that naïve meditators mind wander excessively, as expected. In average their ANN response is +0.24 during five minutes of meditation. Further, only $25 \%$ of their ANN responses fall below +0.02 , which means that in nearly $75 \%$ of the cases they do show positive DMN activity. Actually, some of this group's extreme data reaches +1 , the highest level of DMN activation. As for the second group, it becomes apparent that the inclusion of Ānāpāna dramatically improves the performance. The average of ANN responses drops off to -0.61 and $75 \%$ of the outputs fall below +0.04 . In other words, this group predominantly shows DMN deactivation (negative ANN outputs) during the test. For example, in the worst case, they mind wander up to +0.39 , which is still acceptable compared to their previous meditative peers. As expected, the third group shows in turn the best performance, with lowest DMN activity and least variability in ANN outputs. This means that these subjects were able to keep steady concentration during the task. Accordingly, these subjects never activated the offsetting microphone, since not even in the worst case their mind wandering reached -0.5 (threshold for activation). This is perhaps more noticeable in Fig. 11, where we have plotted the ANN outputs (as a signal) of one group member (randomly chosen) per group.
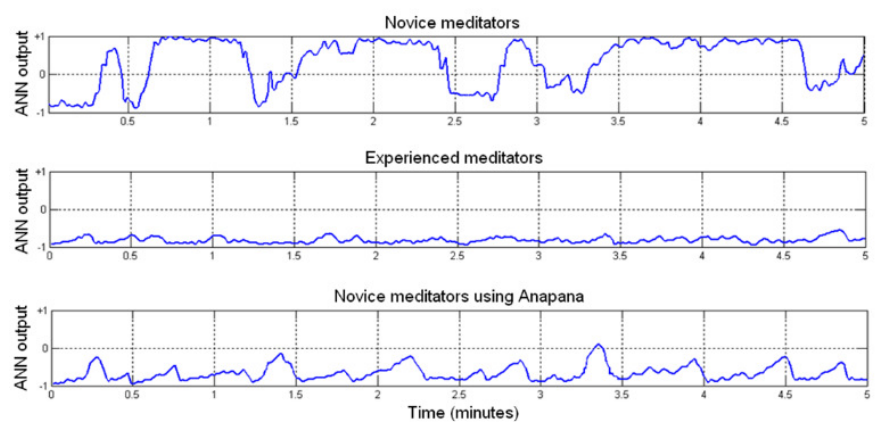

Figure 11. ANN outputs for one subject (randomly chosen) of each group.

In the figure above (bottom signal), we can clearly see how our system effectively prevents attention from dropping off. Every time that DMN activity intensifies (signal rises), a sudden decrease takes place to shape a pick in the signal. This decrease is certainly induced by the offsetting microphone which is triggered once the signal reaches a critical threshold; i.e. -0.5. At this point, the artificially enhanced breathing helps the naïve meditator to fix the attention back on it. In fact, a pattern of picks with high frequency reflects the struggling of the novice meditator, whose mind tends to wander but is constantly inhibited. Yet, they do not mind wander at all. In sharp contrast, experienced mediators exhibit an almost flat curve that can be interpreted as calamity. This also applies to novice meditators without Ānāpāna; they do not register a consistent pattern of picks, for the struggling just fades away when the mind begins to wander. Our assumption is that with time, such induced increase of attention will become natural and the struggling will cease. As it is mostly the case when training a particular skill.

\section{CONCLUSION}

There is absolutely no need to constrain the applicability of Ānāpāna to depression, nor is medical consent/supervision a requirement for it to be used. Due to its portability and easiness, Ānāpāna can be used by anyone seeking to enhance 
his (her) meditation skills at any moment, anywhere. There are scientifically validated benefits of 30-to-40 minutes of meditation a day. Particularly, it has been shown that mindfulness can reshape our brains in the long run (i.e. neuroplasticity). For example, many neuroscientists regard this practice as a natural way to alleviate anxiety, insomnia and stress, to reverse heart disease, and to enhance attention [8]. Both, neuroimaging and encephalography research support that mindfulness slows down the natural age-related cortical decline, and decreases the amygdala's gray matter (associated with fear and anger) [9], [18]. Consequently, we presented here an assistive device to improve the quality of life for the benefit of modern busy society.

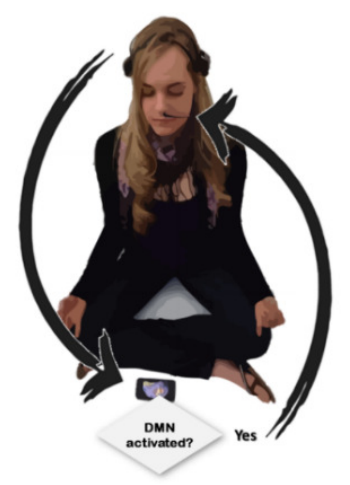

Figure 12. Ānāpāna prototype. Note how this prototype simplifies the framework shown in Fig. 2.

Finally, in Fig. 12 we can clearly see how the framework presented in Fig. 2 can be simplified by the system proposed in this paper (i.e. Ānāpāna). Yet a final prototype is in need of validation with patients. This is, therefore, the focus of our future research. We want first to verify more robustly our hypothesis that artificially enhanced breath prevents mind wandering. Moreover, after that, we would like to prove that our system speeds up the learning of mindfulness and hence, the reduction of depression, anxiety and other mental disorders. In doing so, we need to deal with depressed patients, clinicians, neuroscientist and ethical committees.

\section{ACKNOWLEDGEMENT}

Special thanks to Prof. Olaf Blank from Brain Mind Institute (École Polytechnique Fédérale de Lausanne - EPFL) for his support in this work.

\section{REFERENCES}

[1]. World Health Org. (2010, March) WHO. [Online]. http://www.who.int/mediacentre/factsheets/fs282/en/

[2]. Smallwood, J., Fitzgerald, A., Miles, L. K., \& Phillips, L. H. (2009). Shifting moods, wandering minds: negative moods lead the mind to wander. Emotion, 9(2), 271-276.

[3]. Christoff, K., Gordon, A., Smallwood, J., \& Schooler, J. (2009). Experience sampling during
fMRI reveals default network and executive system contributions to mind wandering. Proceedings of the National Academy of Sciences, 106(21), 8719-8724.

[4]. Brown, K. W., \& Ryan, R. M. (2003). The benefits of being present: mindfulness and its role in psychological well-being. Journal of Personality and Social Psychology, 84(4), 822-848.

[5]. 10-20 system (EEG). (2010, March) Wikipedia. [Online]. 20_system_(EEG)

[6]. [Baldi, P., \& Hornik, K. (1989). Neural networks and principal component analysis: Learning from examples without local minima. Neural Networks, vol. 2, no. 10, pp. 53-58, 1989.

[7]. Emotiv EPOC. (2010, March) EPOC. [Online]. http://emotiv.com/epoc/

[8]. Flook, L., Goldberg, S. B., Pinger, L., Bonus, K., \& Davidson, R. J. (2013). Mindfulness for teachers: A pilot study to assess effects on stress, burnout and teaching efficacy. Mind, Brain and Education, 7, 182-195.

[9]. Lee, H, \& Davidson, R. J. (2012). Amygdalaprefrontal coupling underlies individual differences in emotion regulation. Neuroimage, 62(3), 1575-81. PMCID: PMC3408571.

[10]. Center for investigating healthy minds. (2010, March) [Online]. http://www.investigatinghealthyminds.org/cihmDrDa vidson.html.

[11]. Antoine, L., Lawrence L. G., Nancy B. R., Matthieu R., \& Davidson, R. J. (2004). Long-term meditators self-induce high-amplitude gamma synchrony during mental practice. PNAS 2004101 (46) 16369-16373; published ahead of print November 8, 2004, doi:10.1073/pnas.0407401101.

[12]. Ekman P., Davidson R.J., Ricard M., Wallace A. (2005). Buddhist and psychological perspectives on emotions and well-being. Current Directions in Psychological Science, 14, 59-63.

[13]. Abercrombie H.C., Schaefer S.M., Larson C.L., Oakes T.R., Lindgren K.A., Holden J.E., Davidson R.J. (1998). Metabolic rate in the right amygdala predicts negative affect in depressed patients. NeuroReport, 9, 3301-3307.

[14]. Anand A., Li Y., Wang Y., Wu J., Gao S., Bukhari L., Lowe M.J. (2005). Activity and connectivity of brain mood regulating circuit in depression: A functional magnetic resonance study. Biological Psychiatry, 57, 1079-1088.

[15]. Anderson N.D., Lau M.A., Segal Z.V., Bishop S.R. (2007). Mindfulness-based stress reduction and attentional control. Clinical Psychology \& Psychotherapy, 14, 449-463.

[16]. Austin J.H. (2006). Zen-brain reflections. Cambridge, MA: MIT Press.

[17]. Baer R.A. (2003). Mindfulness training as a clinical intervention: A conceptual and empirical 
review. Clinical Psychology: Science and Practice, $10,125-143$.

[18]. [Banks S.J., Eddy K.T., Angstadt M., Nathan P.J., Phan K.L. (2007). Amygdala-frontal connectivity during emotion-regulation. Social Cognitive and Affective Neuroscience, 2, 303-312.

[19]. Bishop S.R., Lau M., Shapiro S., Carlson L.E., Anderson N.D., Carmody J., Devins G. (2004). Mindfulness: A proposed operational definition. Clinical Psychology: Science and Practice, 11, 230241.

[20]. Brefczynski-Lewis J.A., Lutz A., Schaefer H.S., Levinson D.B., Davidson R.J. (2007). Neural correlates of attentional expertise in long-term meditation practitioners. Proceedings of the National Academy of Sciences of the United States of America, 104, 11483-11488.

[21]. Brewer J.A., Kober H., Worhunsky P.D., Tang Y.-Y., Gray J.R., Weber J. (2011). Mental training reveals differences in default mode network activation and functional connectivity. Manuscript submitted for publication.

[22]. Brown K.W., Ryan R.M. (2003). The benefits of being present: Mindfulness and its role in psychological well-being. Journal of Personality and Social Psychology, 84, 822-848.

[23]. Buckner R.L., Andrews-Hanna J.R., Schacter D.L. (2008). The brain's default network: Anatomy, function, and relevance to disease. Annals of the New York Academy of Sciences, 1124, 1-38.

[24]. Carmody J., Baer R.A. (2008). Relationships between mindfulness practice and levels of mindfulness, medical and psychological symptoms and well-being in a mindfulness-based stress reduction program. Journal of Behavioral Medicine, $31,23-33$.

[25]. Carmody J., Baer R.A., Lykins E.L.B., Olendzki N. (2009). An empirical study of the mechanisms of mindfulness in a mindfulness-based stress reduction program. Journal of Clinical Psychology, 65, 613-626.

[26]. Chiesa A., Serretti A. (2009). Mindfulnessbased stress reduction for stress management in healthy people: A review and meta-analysis. Journal of Alternative and Complementary Medicine, 15, 593-600.

[27]. Farb N.A.S., Segal Z.V., Mayberg H., Bean J., McKeon D., Fatima Z., Anderson A.K. (2007). Attending to the present: Mindfulness meditation reveals distinct neural modes of self-reference. Social Cognitive and Affective Neuroscience, 2, 313-322.

[28]. Goldin P.R., Gross J.J. (2010). Effects of mindfulness-based stress reduction (MBSR) on emotion regulation in social anxiety disorder. Emotion, 10, 83-91.

[29]. Hofmann S.G., Sawyer A.T., Witt A.A., Oh D. (2010). The effect of mindfulness-based therapy on anxiety and depression: A meta-analytic review. Journal of Consulting and Clinical Psychology, 78, 169-183.

[30]. Jang J.H., Jung W.H., Kang D.H., Byun M.S., Kwon S.J., Choi C.H., Kwon J.S. (2010). Increased default mode network connectivity associated with meditation. Neuroscience Letters, 487, 358-362.

[31]. Pizzagalli D. (2010). Frontocingulate Dysfunction in Depression: Toward Biomarkers of Treatment Response Neuropsychopharmacology. 36(1): 183-206. Published online 2010 September 22. doi: $10.1038 / n p p .2010 .166$.

[32]. Mayberg HS, Lozano AM, Voon V, McNeely HE, Seminowicz D, Hamani C, Schwalb JM, Kennedy SH. (2005). Deep brain stimulation for treatment-resistant depression. Neuron. 2005 Mar 3; 45(5):651-60.

[33]. Araujo, D. (20014).The Therapeutic Potentials of Ayahuasca in the Treatment of Depression. Journal of Psychoactive Drugs Volume 30, Issue 4, Special Issue: Therapeutic Use of Hallucinogens.

[34]. H. Larochelle, Y. Bengio, J. Louradour and P. Lamblin, "Exploring Strategies for Training Deep Neural Networks," Journal of Machine Learning Research, vol. 20, no. 1, pp. 1-40, 2009.

[35]. A. Krizhevsky and G. Hinton, "Using Very Deep Autoencoders for Content-Based Image Retrieval," in European Symposium on Artificial Neural Networks ESANN, Bruges, 2011.

[36]. R. Sarikaya and G. Hinton, "Deep Belief Nets for Natural Language Call-Routing," in International Conference on Acoustics, Speech, and Signal Processing ICASSP, New York, 2011.

[37]. Y. LeCun, L. Bottou, Y. Bengio and P. Haffner, "Gradient-based learning applied to document recognition," Proceedings of the IEEE, vol. 86, no. 11, p. 2278-2324, 1998.

[38]. S. University, "UFLDL Tutorial," Stanford University, 11 April 2011. [Online]. Available: http://ufldl.stanford.edu/wiki/index.php/UFLDL_Tut orial. [Accessed 30 January 2013].

[39]. Pun, T. (2012).The impact of denoising on independent component analysis of functional magnetic resonance imaging data. Journal of Neuroscience Methods 213 (2013) 105-122.

[40]. Gomez, Juan; et al. Eterno de resplandor de una mente sin recuerdos: si me dejas te suprimo. Scientia et Technica. Universidad Tecnológica de Pereira. ISSN 0122-170. No 48, Agosto de 2011. 